\title{
A SIMPLIFIED VERSION OF SPITZER'S FORMULA FOR SEMICONTINUOUS AND ALMOST SEMICONTINUOUS PROCESSES
}

UDC 519.21

\author{
D. V. GUSAK
}

\begin{abstract}
Let $\{\xi(t), \zeta(0)=0, t \geq 0\}$ be a process with stationary independent increments. We establish simplified versions of relations for spectral functions in Spitzer's formulas in terms of the exponential function whose index is determined by the corresponding root of Lundberg's equation for the case where $\xi(t)$ is a semicontinuous or almost semicontinuous process.
\end{abstract}

The characteristic functions of extremal values of an arbitrary homogeneous process $\xi(t), t \geq 0, \xi(0)=0$, with independent increments are determined by the Spitzer formulas (see [1, 2]). Namely, if

$$
\xi^{+}(t)=\sup _{0 \leq t^{\prime} \leq t} \xi\left(t^{\prime}\right), \quad \xi^{-}(t)=\inf _{0 \leq t^{\prime} \leq t} \xi\left(t^{\prime}\right),
$$

then the Spitzer formulas imply that the integral transforms of the characteristic functions $\mathrm{E} e^{i \alpha \xi^{ \pm}(t)}$ are the characteristic functions of infinitely divisible distributions with spectral functions $N_{s}^{ \pm}(x)( \pm x>0)$. The functions $N_{s}^{ \pm}(x)$ are rather complicated integral transforms of the distributions of the positive and negative parts of $\xi(t)$.

The spectral functions $N_{s}^{ \pm}(x)$ become much simpler for semicontinuous and almost semicontinuous processes and this results in simpler expressions for the characteristic functions of $\xi^{ \pm}\left(\theta_{s}\right)$. This simplification of the expressions for $N_{s}^{ \pm}(x)$ is due to the duality between the distributions of $\xi^{+}(t)$ and $\tau^{+}(x)=\inf \{t: \xi(t)>x\}, x>0$.

If $\xi(t)$ is an arbitrary homogeneous process with independent increments, then $\theta_{s}$ has the exponential distribution, that is, $\mathrm{P}\left\{\theta_{s}>t\right\}=e^{-s t}, s>0$. Therefore the characteristic functions

$$
\varphi_{ \pm}(s, \alpha)=: \mathrm{E} e^{i \alpha \xi^{+}\left(\theta_{s}\right)}=s \int_{0}^{\infty} e^{-s t} \mathrm{E} e^{i \alpha \xi^{ \pm}(t)} d t
$$

are determined by the following relations (called Spitzer's formulas):

$$
\varphi_{ \pm}(s, \alpha)=\exp \left\{ \pm \int_{0}^{ \pm \infty}\left(e^{i \alpha x}-1\right) d N_{s}^{ \pm}(x)\right\}
$$

where

$$
\begin{array}{r}
N_{s}^{+}(x)=-\int_{0}^{\infty} e^{-s t} t^{-1} \mathrm{P}\{\xi(t)>x\} d t, \quad x>0, \\
N_{s}^{-}(x)=\int_{0}^{\infty} e^{-s t} t^{-1} \mathrm{P}\{\xi(t)<x\} d t,
\end{array}
$$

2000 Mathematics Subject Classification. Primary 60G50; Secondary 60K10.

Key words and phrases. Semicontinuous and almost semicontinuous processes with independent increments, Spitzer's formula, Lundberg's equation, Spitzer's spectral functions. 
(see 3], Theorem 1.6 and equalities (1.64)-(1.65) therein). The main aim of this paper is to study some cases where relations $(2)$ for $N_{s}^{ \pm}(x)$ can be simplified to some extent.

Spitzer's formulas (1.65)-(1.66) given in [3] become much simpler for semicontinuous and almost semicontinuous processes if one uses the results of $\S \S 3.1-3.2$ in $\underline{3}$. We deal with the Spitzer spectral function $N_{s}^{+}(x)$ in Section 1 for upper semicontinuous processes and represent a simplified version of the Spitzer formula for $\xi^{+}\left(\theta_{s}\right)$. A similar result holds for upper almost semicontinuous processes as well. In this case, the result is first proved for the conditional moment-generating function $\mathrm{P}\left\{\xi^{+}\left(\theta_{s}\right)>0\right\}=q_{+}(s)>0$, namely for

$$
\hat{\varphi}_{+}(s, i z)=: \mathrm{E}\left[e^{-z \xi^{+}\left(\theta_{s}\right)} \mid \xi^{+}\left(\theta_{s}\right)>0\right]=\mathrm{E}\left[e^{-z \xi^{+}\left(\theta_{s}\right)}, \xi^{+}\left(\theta_{s}\right)>0\right] q_{+}^{-1}(s),
$$

and then for the unconditional moment-generating function $\varphi_{+}(s, i z)$.

In Section 2, we obtain a relationship between the minimum $\xi^{-}(t)$ of the underlying process and the distribution of the negative part of $\xi(t)$ in the case of upper semicontinuous processes. It is worthwhile mentioning that relations (1)-(2) for $N_{s}^{-}(x)$ are not used when deriving this relationship.

\section{A Simplified FORM OF THE SPECTRAL FUnCtion $N_{s}^{+}(x)$ FOR UPPER SEMICONTINUOUS AND UPPER ALMOST SEMICONTINUOUS PROCESSES}

Let $\xi(t)$ be a monotone upper semicontinuous process with negative jumps, let

$$
\int_{-1}^{0} x^{2} \Pi(d x)<\infty
$$

and let the cumulant of the process be given by

$$
\psi_{1}(\alpha)=i \alpha a^{\prime}-\frac{\sigma^{2} \alpha^{2}}{2}+\int_{-\infty}^{0}\left(e^{i \alpha x}-1-i \alpha x \mathbb{1}_{|x| \leq 1}\right) \Pi(d x)
$$

where

and

$$
a=a^{\prime}-\int_{-\infty}^{0} x \Pi(d x)>0
$$

$$
\sigma^{2}=0, \quad \int_{-1}^{0}|x| \Pi(d x)>0 .
$$

The duality between the distributions of $\xi(t)>0$ and $\tau^{+}(x)$ is obtained independently and almost simultaneously by Keilson [4], Zolotarev [5], and Borovkov [6] for the processes $\xi(t)$ whose cumulant is given by (10) (see (3.70) in [3] for $F(t, x)=\mathrm{P}\{\xi(t)<x\}$ ). This duality is written as

$$
\frac{\partial}{\partial x} F(t, x)=t x^{-1} \frac{\partial}{\partial t} \mathrm{P}\left\{\tau^{+}(x)<t\right\}, \quad x>0 .
$$

A similar relationship between the conditional distribution of the first passage time $\left\{\tau^{+}(x) / \tau^{+}(0)<t\right\}$ and the distribution of $\xi(t)$ is obtained in [3] (see equality (5.45) therein), namely

$$
\frac{\partial}{\partial x} F(t, x)=t x^{-1} \frac{\partial}{\partial t} \mathrm{P}\left\{\tau^{+}(x)<t / \tau^{+}(0)<t\right\}, \quad x>0,
$$

for upper almost semicontinuous processes with exponential positive jumps and the cumulant

$$
\begin{gathered}
\psi_{2}(\alpha)=i \alpha a+\lambda_{1} \frac{i \alpha}{c-i \alpha}+\int_{-\infty}^{0}\left(e^{i \alpha x}-1\right) \Pi(d x), \\
f(z)=\mathrm{E}\left[e^{-z \xi} / \xi>0\right]=\frac{c}{c-i \alpha}, \quad \lambda_{1}, c>0, a \leq 0 .
\end{gathered}
$$


Using (4) and (3) we establish simple relations for the derivatives of the spectral functions

if $m=\mathrm{E} \xi(1)<0$. Put

$$
\frac{\partial}{\partial x} N_{s}^{+}(x), \quad \frac{\partial}{\partial x} N_{0}^{+}(x)
$$

$$
P(s, x)=\mathrm{P}\left\{\xi\left(\theta_{s}\right)<x\right\}, \quad \bar{P}(s, x)=1-P(s, x), \quad P_{ \pm}(s, x)=\mathrm{P}\left\{\xi^{ \pm}\left(\theta_{s}\right)<x\right\} .
$$

Theorem 1. Let a process $\xi(t)$ have the cumulant given by (11). Then the momentgenerating function of $\xi^{+}\left(\theta_{s}\right)$ is determined by the canonical representation (1.65) in [3] where the derivative of the spectral function $N_{s}^{+}(x)$ is of the following form:

$$
\begin{gathered}
\varphi_{+}(s, i z)=\exp \left\{\int_{0}^{\infty}\left(e^{-z x}-1\right) d N_{s}^{+}(x)\right\}, \\
\frac{\partial}{\partial x} N_{s}^{+}(x)=x^{-1} \mathrm{P}\left\{\xi^{+}\left(\theta_{s}\right)>x\right\}=x^{-1} e^{-\rho_{+}(s) x}, \quad x>0,
\end{gathered}
$$

and where $\rho_{+}(s)$ is the root of the Lundberg equation $k_{1}(r)=\psi_{1}(-i r)=s$ :

$$
\rho_{+}(s)= \begin{cases}P^{\prime}(s, 0) \bar{P}^{-1}(s, 0), & \sigma \geq 0, \int_{-1}^{0} x^{2} \Pi(d x)<\infty, \\ s\left(a p_{-}(s)\right)^{-1}, & \sigma=0, \int_{-1}^{0}|x| \Pi(d x)<\infty .\end{cases}
$$

If $m=\mathrm{E} \xi(1)<0$, then $\rho_{+}(s) \rightarrow \rho_{+}>0, N_{s}^{+}(x) \rightarrow N_{0}^{+}, x>0$, and

$$
\frac{\partial}{\partial x} N_{0}(x)=x^{-1} e^{-\rho_{+}(s) x}, \quad\left|N_{0}(x)\right|=\int_{x}^{\infty} y^{-1} e^{-\rho_{+} y} d y, \quad x>0 .
$$

Proof. According to (2) the Spitzer spectral function

$$
\left|N_{s}^{+}(x)\right|=\int_{0}^{\infty} e^{-s t} t^{-1} \int_{x}^{\infty} \frac{\partial}{\partial y} F(t, y) d y d t
$$

is reduced to

$$
\begin{aligned}
\left|N_{s}^{+}(x)\right| & =\int_{0}^{\infty} e^{-s t} t^{-1} \int_{x}^{\infty} t y^{-1} \frac{\partial}{\partial t} \mathrm{P}\left\{\tau^{+}(y)<t\right\} d y d t \\
& =\int_{0}^{\infty} e^{-s t} \frac{\partial}{\partial t} \int_{x}^{\infty} \mathrm{P}\left\{\tau^{+}(y)<t\right\} d \ln y d t .
\end{aligned}
$$

Integrating by parts in the integral with respect to $y$ and then in the integral with respect to $t$ we find

$$
\begin{aligned}
\left|N_{s}^{+}(x)\right| & =\int_{0}^{\infty} e^{-s t} \frac{\partial}{\partial t}\left[\int_{x}^{\infty} \ln y d \mathrm{P}\left\{\xi^{+}(t)<y\right\}-\ln x \mathrm{P}\left\{\xi^{+}(t)>x\right\}\right] d t \\
& =s \int_{0}^{\infty} e^{-s t}\left[\ln x \mathrm{P}\left\{\xi^{+}(t)>x\right\}-\mathrm{E} \ln \xi^{+}(t) \mathbb{1}_{\xi^{+}(t)>x}\right] d x \\
& =\ln x \bar{P}_{+}(s, x)-\mathrm{E} \ln \xi^{+}\left(\theta_{s}\right) \mathbb{1}_{\xi^{+}\left(\theta_{s}\right)>x}=\int_{x}^{\infty} \ln \frac{x}{y} d P_{+}(s, y)
\end{aligned}
$$

for $x>0$. Thus the derivative of the function $N_{s}^{+}(x)$ is expressed in terms of

$$
\bar{P}_{+}(s, x)=1-P_{+}(s, x)
$$

as follows:

$$
\frac{\partial}{\partial x} N_{s}^{+}(x)=\int_{x}^{\infty}\left(\ln \frac{x}{y}\right)^{\prime} d P_{+}(s, y)=\frac{1}{x} \bar{P}_{+}(s, x), \quad x>0 .
$$

This implies relation (5), since $\bar{P}_{+}(s, x)=e^{-\rho_{+}(s) x}, x>0$, for upper semicontinuous processes. If $m<0$ and $s \rightarrow 0$, then equality (5) implies (6). 
Theorem 2. Let the cumulant of a process be given by (44). Then the conditional moment-generating function of $\xi^{+}\left(\theta_{s}\right)>0$ is determined by the following relation:

$$
\hat{\varphi}_{+}(s, i z)=: \mathrm{E}\left[e^{-z \xi^{+}\left(\theta_{s}\right)} / \xi^{+}\left(\theta_{s}\right)>0\right]=\frac{\rho_{+}(s)}{\rho_{+}(s)+z},
$$

where $\rho_{+}(s)=c p_{+}(s)$ is the root of the Lundberg equation $k_{2}\left(\rho_{+}(s)\right)=s$,

$$
p_{+}(s) p_{-}(s)=s(s+\lambda)^{-1}
$$

for the case of $\int_{-\infty}^{0} \Pi(d x)<\infty$. Moreover $\hat{\varphi}_{+}(s, i z)$ admits the canonical representation with the spectral function $\hat{N}_{s}(x), x>0$, namely

$$
\hat{\varphi}_{+}(s, i z)=\exp \left\{\int_{0}^{\infty}\left(e^{-z x}-1\right) d \hat{N}_{s}(x)\right\}
$$

where

$$
\left|\hat{N}_{s}(x)\right|=\frac{1}{q_{+}(s)} \int_{x}^{\infty} y^{-1} \bar{P}_{+}(s, y) d y, \quad p_{ \pm}(s)=\mathrm{P}\left\{\xi^{ \pm}\left(\theta_{s}\right)=0\right\},
$$

$\hat{N}_{s}^{\prime}(x)=\frac{\partial}{\partial x} \hat{N}_{s}(x)=\frac{1}{q_{+}(s) x} P_{+}(s, x)=x^{-1} e^{-\rho_{+}(s) x}, \quad q_{+}(s)=1-p_{+}(s), \quad x>0$.

If $m<0$, then $\rho_{+}(s) \underset{s \rightarrow 0}{\rightarrow} \rho_{+}=c p_{+}$and $\hat{N}_{s}(x) \underset{s \rightarrow 0}{\rightarrow} \hat{N}_{0}(x)$, where $p_{+}=\mathrm{P}\left\{\xi^{ \pm}=0\right\}$,

$$
\hat{N}_{0}^{\prime}(x)=\frac{1}{q_{+} x} \mathrm{P}\left\{\xi^{+}>x\right\}=x^{-1} e^{\rho_{+} x}, \quad x>0 .
$$

Proof. According to (3), the spectral function $\hat{N}_{s}(x)$ in (77) can be rewritten as follows:

$$
\begin{aligned}
\left|\hat{N}_{s}(x)\right| & =\int_{0}^{\infty} e^{-s t} \frac{\partial}{\partial t}\left(\int_{x}^{\infty} \mathrm{P}\left\{\xi^{+}(t)>y / \xi^{+}(t)>0\right\} y^{-1} d y\right) d t \\
& =\int_{0}^{\infty} e^{-s t} \frac{\partial}{\partial t}\left(\int_{x}^{\infty} \mathrm{P}\left\{\xi^{+}(t)>y / \xi^{+}(t)>0\right\} d \ln y\right) d t .
\end{aligned}
$$

Integrating by parts in the integral with respect to $y$ and then in the integral with respect to $t$ we find

$$
\begin{aligned}
\left|\hat{N}_{s}(x)\right| & =\int_{0}^{\infty} e^{-s t} \frac{\partial}{\partial t} \frac{1}{\mathrm{P}\left\{\xi^{+}(t)>0\right\}}\left[\mathrm{E} \ln \xi^{+}(t) \mathbb{1}_{\xi^{+}(t)>x}-\ln x \mathrm{P}\left\{\xi^{+}(t)>x\right\}\right] d t \\
& =\int_{0}^{\infty} \frac{s e^{-s t}}{\mathrm{P}\left\{\xi^{+}(t)>0\right\}}\left[\mathrm{E} \ln \xi^{+}(t) \mathbb{1}_{\xi^{+}(t)>x}-\ln x \mathrm{P}\left\{\xi^{+}(t)>x\right\}\right] d t \\
& =\frac{1}{\bar{P}_{+}(s, 0)} \int_{x}^{\infty}(\ln x-\ln y) d P_{+}(s, y), \quad x>0, \bar{P}_{+}(s, 0)=q_{+}(s) .
\end{aligned}
$$

Thus the derivative of $\hat{N}_{s}(x)$ becomes

$$
\hat{N}_{s}^{\prime}(x)=\frac{1}{q_{+}(s) x} \bar{P}_{+}(s, x), \quad x>0 .
$$

This implies (8), since

$$
\bar{P}_{+}(s, x)=q_{+}(s) e^{-\rho_{+}(s) x}, \quad x>0,
$$

for upper almost semicontinuous processes.

Passing to the limit in (8) as $s \rightarrow 0$ we get equality (9) if $m<0$. 
Theorem 3. According to relation (3.87) in [3], the unconditional moment-generating function of $\xi^{+}\left(\theta_{s}\right) \geq 0$ is given by

$$
\varphi_{+}(s, i z)=\frac{p_{+}(s)(c+z)}{\rho_{+}(s)+z}=\hat{\varphi}_{+}(s, z) f^{-1}(z), \quad f(z)=\frac{c}{c+z} .
$$

Moreover, the unconditional moment-generating function admits a canonical representation similar to (5), where the derivative of the spectral function $N_{s}(x)$ is equal to

$$
N_{s}^{\prime}(x)=\hat{N}_{s}^{\prime}(x)-x^{-1} e^{-c x}=x^{-1} e^{-c x}\left(e^{q_{+}(s)}-1\right), \quad x>0 .
$$

If $m<0$, then

$$
N_{s}^{\prime}(x) \underset{s \rightarrow 0}{\longrightarrow} N_{0}^{\prime}(x)=x^{-1} e^{-c x}\left(e^{q_{+} x}-1\right), \quad x>0 .
$$

Proof. Equalities (10) imply that

$$
\ln \varphi_{+}(s, i z)=\int_{0}^{\infty}\left(e^{-z x}-1\right) d N_{s}(x)=\ln \hat{\varphi}_{+}(s, i z)-\ln f(z),
$$

where $\ln f(z)$ can be represented in the form of the Frullani integral as follows:

$$
\ln f(z)=\ln \frac{c}{c+z}=\int_{0}^{\infty} x^{-1}\left(e^{-z x}-1\right) e^{-c x} d x
$$

Taking the logarithm in (7), substituting the result to equality (13), and then using (14), we obtain equalities (11). Passing to the limit in (11) as $s \rightarrow 0$ we prove (12) in the case of $m<0$.

Theorems 13 yield the following result.

Corollary 1. Averaged with respect to $t$, the tails of the distribution of $\xi(t)>0$ are determined for $x>0$ by the densities

$$
\begin{aligned}
-\frac{\partial}{\partial x} & \left(\int_{0}^{\infty} \bar{F}(t, x) e^{-s t} d \ln t\right) \\
& = \begin{cases}x^{-1} e^{-\rho_{+}(s) x}, & \text { if the cumulant of } \xi(t) \text { is given by (1) }, \\
x^{-1} e^{-c x}\left(e^{-c q_{+}(s) x}-1\right), & \text { if the cumulant of } \xi(t) \text { is given by (4). }\end{cases}
\end{aligned}
$$

If $m<0$, then

$$
\begin{aligned}
-\frac{\partial}{\partial x} & \left(\int_{0}^{\infty} \bar{F}(t, x) d \ln t\right)=-\frac{\partial}{\partial x} \int_{-\infty}^{\infty} \bar{F}\left(e^{y}, x\right) d y \\
& = \begin{cases}x^{-1} e^{-\rho_{+} x}, & \text { if the cumulant of } \xi(t) \text { is given by (1) } \\
x^{-1} e^{-c x}\left(e^{-c q_{+} x}-1\right), & \text { if the cumulant of } \xi(t) \text { is given by (4) }\end{cases}
\end{aligned}
$$

for $x>0$.

Example 1. Consider the process introduced in Example 3.3 in [3], namely

$$
\xi(t)=w(t)-t+\sum_{k \leq \nu(t)} \xi_{k}, \quad \mathrm{E} e^{-z \xi_{k}}=f(z)=\frac{c}{c+z}, \quad c>0,
$$

where $\nu(t)$ is a Poisson process with intensity $\lambda>0$ and where $w(t)$ is a standard Wiener process. We find the derivative of the spectral functions $\frac{\partial}{\partial x} N_{s}(x)$ and $\frac{\partial}{\partial x} N_{0}(x)$ for $c=6$ and $\lambda=\frac{5}{8}$.

It is easy to prove that the cumulant of $\xi(t)$,

$$
\psi(\alpha)=-i \alpha-\frac{\alpha^{2}}{2}+\lambda \frac{i \alpha}{c-i \alpha},
$$


after the change $i \alpha=r$, is expressed in terms of $m=\lambda c^{-1}-1$, namely

$$
k(r)=\psi(-i r)=\frac{-r^{3}-(c+2) r^{2}-2 m c r}{2(c-r)} .
$$

In this case, the Lundberg equation $k(r)=s$ reduces to the cubic equation

$$
r^{3}-(2+c) r^{2}-2(m c+s) r+2 c s=0, \quad s>0,
$$

possessing the three roots $r_{1}(s)=-\rho_{-}(s)<0,0<r_{2}(s)<r_{3}(s)$. The first root defines the moment-generating function of $\xi^{-}\left(\theta_{s}\right)$ :

$$
\varphi_{-}(s, \alpha)=\frac{\rho_{-}(s)}{\rho_{-}(s)+i \alpha} .
$$

The positive roots define the moment-generating function of $\xi^{+}\left(\theta_{s}\right)$ :

$$
\varphi_{+}(s, i z)=r_{2}(s)\left(r_{2}(s)+z\right)^{-1} r_{3}(s)\left(r_{3}(s)+z\right)^{-1} f^{-1}(z) .
$$

The logarithms of factors in (21) can be represented in the form of the Frullani integrals,

$$
\ln \frac{r_{k}(s)}{r_{k}(s)+z}=\int_{0}^{\infty} e^{-r_{k}(s) x}\left(e^{-x z}-1\right) x^{-1} d x, \quad k=2,3 .
$$

Taking the logarithms of both sides of relation (21) and then substituting (14) and (22), we obtain

$$
\frac{\partial}{\partial x} N_{s}(x)=x^{-1}\left(e^{-r_{2}(s) x}+e^{-r_{3}(s) x}-e^{-c x}\right), \quad x>0 .
$$

If $m<0$ and $\lambda<c$, then $r_{1}(s) \rightarrow 0$ and $r_{2,3}(s) \rightarrow r_{2,3}>0$ as $s \rightarrow 0$. Hence

$$
\frac{\partial}{\partial x} N_{0}(x)=x^{-1}\left(e^{-r_{2} x}+e^{-r_{3} x}-e^{-c x}\right), \quad x>0 .
$$

Substituting $\lambda=\frac{5}{8}$ and $c=6$ we find that $D_{0}=9, r_{2}=2.5<r_{3}=5.5$, and

$$
\frac{\partial}{\partial x} N_{0}(x)=x^{-1} e^{-6 x}\left(e^{2.5 x}+e^{0.5 x}-1\right), \quad x>0 .
$$

This example shows that the number of exponents in $\frac{\partial}{\partial x} N_{s}(x)$ and $\frac{\partial}{\partial x} N_{0}(x)$ increases if the roots of the Lundberg equation become larger. One of the exponents is determined by the exponential distribution of jumps of $\xi(t)$ with parameter $c=6$. An analogous result holds if positive jumps of $\xi(t)$ have the Erlang distribution $E(n)$ of order $n \geq 2$ and if $\xi(t)$ is a lower semicontinuous or almost semicontinuous process.

Example 2. Let $\xi(t)=S(t)-t$ be the process considered in Example 5.1 in 3 for which the characteristic function of jumps is of the type $E(2)$; here

$$
\begin{gathered}
S(t)=\sum_{k \leq \nu(t)} \xi_{k}, \\
\varphi(\alpha)=\mathrm{E} e^{i \alpha \xi_{k}}=\frac{1}{2}\left(\frac{3}{3-i \alpha}+\frac{7}{7-i \alpha}\right), \quad m=-\frac{2}{7} .
\end{gathered}
$$

Our aim is to find the derivatives $\frac{\partial}{\partial x} N_{s}(x)$ and $\frac{\partial}{\partial x} N_{0}(x), x>0$.

Similarly to the preceding example, the Lundberg equation reduces to a cubic equation. This equation for $s=0$ is given by

$$
r^{3}-7 r^{2}+6 r=0
$$


and has the roots $r_{1}=-\rho_{-}=0, r_{2}=R_{+}=1$, and $r_{3}=6$. If $s>0$, then the Lundberg equation has the following roots:

$$
\begin{gathered}
r_{1}(s)=-\rho_{-}(s), \quad 0<r_{2}(s)=R_{+}(s)<r_{3}(s), \\
r_{1}(s) \underset{s \rightarrow 0}{\longrightarrow} 0, \quad r_{2,3}(s) \underset{s \rightarrow 0}{\longrightarrow} r_{2,3}>0,
\end{gathered}
$$

and these roots determine the distributions of $\xi^{ \pm}\left(\theta_{s}\right)$ :

$$
\varphi_{-}(s, \alpha)=\frac{\rho_{-}(s)}{\rho_{-}(s)+i \alpha}, \quad \varphi_{+}(s, i z)=\frac{s}{\rho_{-}(s)} \frac{(3+z)(7+z)}{\left(r_{2}(s)+z\right)\left(r_{3}(s)+z\right)} .
$$

The moment-generating function of $\xi^{+}\left(\theta_{s}\right)$ reduces to the form

$$
\varphi_{+}(s, i z)=\frac{r_{2}(s)}{r_{2}(s)+z} f_{1}^{-1}(z) f_{2}^{-1}(z),
$$

where $f_{k}=\frac{c_{k}}{c_{k}+z}, c_{1}=3$, and $c_{2}=7$. Thus

$$
\begin{aligned}
\ln \varphi_{+}(s, i z) & =\int_{0}^{\infty}\left(e^{-z x}-1\right) d N_{s}(x) \\
& =\ln \frac{r_{2}(s)}{r_{2}(s)+z}+\ln \frac{r_{3}(s)}{r_{3}(s)+z}-\ln f_{1}(z)-\ln f_{2}(z) .
\end{aligned}
$$

Considering the representation of the logarithms in (26) via the Frullani integrals, we prove that

$$
\frac{\partial}{\partial x} N_{s}(x)=x^{-1}\left(e^{-r_{2}(s) x}+e^{-r_{3}(s) x}-e^{-7 x}\right), \quad x>0 .
$$

Since $m=-\frac{2}{7}<0, \ln \mathrm{E} e^{-z \xi^{+}}$is obtained from the relation

$$
\begin{gathered}
\ln \varphi_{+}(i z)=\int_{0}^{\infty}\left(e^{-z x}-1\right) \frac{\partial}{\partial x} N_{0}^{+}(x) d x, \\
\frac{\partial}{\partial x} N_{0}^{+}(x)=x^{-1}\left(e^{-x}+e^{-6 x}-e^{-3 x}-e^{-7 x}\right), \quad x>0 .
\end{gathered}
$$

Two exponents in (28) are explained by the exponential distributions that determine the distribution $E(2)$ of claims $\xi_{k}$ with indices $c_{1}=3$ and $c_{2}=7$.

\section{A formula for the Distribution of $\xi^{-}(t)$ In terms of the Distribution OF $\xi(t)<0$ FOR UPPER SEMICONTINUOUS PROCESSES}

Let $\xi(t)$ be a process whose cumulant is given by (11). In this section, we show that the distribution of $\xi^{-}(t)$ can explicitly be expressed in a simpler form in terms of the distribution of $\xi(t)<0$ for the following two particular cases:

1) $\xi(t)$ is of bounded variation; $\sigma^{2}=0 ; a>0$; and $\int_{-1}^{0}|x| \Pi(d x)<0$;

2) $\xi(t)$ is of unbounded variation.

In what follows we will use the following notation:

$$
\begin{gathered}
\tilde{P}_{>}(s, \alpha)=\int_{R^{+}} e^{i \alpha x} \bar{P}(s, x) d x, \quad \tilde{P}_{<}(s, \alpha)=\int_{R^{-}} e^{i \alpha x} P(s, x) d x \\
\tilde{\Phi}_{-}(s, \alpha)=\int_{R^{-}} P_{-}(s, x) e^{i \alpha x} d x, \quad \tilde{\Phi}_{+}(s, \alpha)=\int_{R^{+}} \bar{P}_{+}(s, x) e^{i \alpha x} d x \\
\mathrm{E}_{ \pm} \xi(t)=\mathrm{E} \xi(t) \mathbb{1}_{ \pm \xi(t)>0}= \pm \int_{R^{ \pm}} \mathrm{P}\{ \pm \xi(t)> \pm x\} d x \\
\mathrm{E}_{+} \xi(t)=\int_{R^{+}} \bar{F}(t, x) d x, \quad \mathrm{E}_{-} \xi(t)=-\int_{R^{-}} F(t, x) d x .
\end{gathered}
$$


Theorem 4. Let $\xi(t)$ be an upper semicontinuous process whose cumulant is given by (10). In the case 1 mentioned above put $a=a^{\prime}-\int_{-1}^{0} x \Pi(d x)>0$. Then

$$
\begin{aligned}
& \tilde{\Phi}_{-}(s, \alpha)=a s^{-1} p_{-}(s) \int_{-\infty}^{0} e^{i \alpha x} d P(s, x)+\tilde{P}_{<}(s, \alpha), \\
& \mathrm{P}\left\{\xi^{-}(t)<x\right\}=F_{-}(t, x) \\
&=F(t, x)+a \int_{0}^{t} \mathrm{P}\left\{\xi^{-}(y)=0\right\} \frac{\partial}{\partial x} F(t-y, x) d y, \quad x<0 .
\end{aligned}
$$

If $m<0$, then $\mathrm{E}_{+} \xi(t)+\mathrm{E}_{-} \xi(t)=m t$ and

$$
\mathrm{P}\left\{\xi^{-}(t)=0\right\}=(a t)^{-1} \mathrm{E}_{+} \xi(t)=(a t)^{-1} \int_{0}^{\infty} \bar{F}(t, x) d x
$$

In both cases 1 and 2 mentioned above we have

$$
\begin{gathered}
\tilde{\Phi}_{-}(s, \alpha)=\mathrm{E} \xi^{+}\left(\theta_{s}\right) \int_{-\infty}^{0} e^{i \alpha x} d P(s, x)+\tilde{P}_{<}(s, \alpha), \\
F_{-}(t, x)=F(t, x)+\int_{0}^{t} \frac{\partial}{\partial x} F(t-y, x) d \mathrm{E} \xi^{+}(y) \\
=F(t, x)+\int_{0}^{t} y^{-1} \mathrm{E}_{+} \xi(y) \frac{\partial}{\partial x} F(t-y, x) d y, \quad x<0 .
\end{gathered}
$$

Proof. We use notation (29). Integrating by parts we prove that

$$
\varphi(s, \alpha)=1+i \alpha\left(\tilde{P}_{>}(s, \alpha)-\tilde{P}_{<}(s, \alpha)\right), \quad \varphi_{-}(s, \alpha)-1=-i \alpha \tilde{\Phi}_{-}(s, \alpha) .
$$

Taking into account (35), we conclude from the main factorization equality (see Theorems 1.16 and 3.1 in [3]) that in both cases

$$
\rho_{+}(s)\left(\tilde{P}_{>}(s, \alpha)-\tilde{P}_{<}(s, \alpha)\right)=\varphi(s, \alpha)-\rho_{+}(s) \tilde{\Phi}_{-}(s, \alpha) .
$$

Applying the projection operator $[\cdot]_{-}$we get

$$
\left[\int_{-\infty}^{\infty} e^{i \alpha x} G(x) d x\right]_{ \pm}=\int_{R^{ \pm}} e^{i \alpha x} G(x) d x
$$

whence we obtain $\tilde{\Phi}_{-}(s, \alpha)$, namely

$$
\tilde{\Phi}_{-}(s, \alpha)=\rho_{+}^{-1}(s) \int_{-\infty}^{0} e^{i \alpha x} d P(s, x)+\tilde{P}_{<}(s, \alpha) .
$$

Note that according to Corollary 3.1 in [3],

$$
p_{-}(s)=\frac{s}{a \rho_{+}(s)}, \quad \rho_{+}^{-1}(s)=\frac{a p_{-}(s)}{s}
$$

in the case 1 mentioned above. Thus (36) implies (30). Inverting (30) with respect to $s$ and $\alpha$ we prove (31).

To prove (32) we note that

$$
\mathrm{E} \xi^{+}\left(\theta_{s}\right)=-\left.\varphi_{+}^{\prime}(s, i z)\right|_{z=0}=\rho_{+}^{-1}(s)=\int_{0}^{\infty} \mathrm{P}\left\{\xi^{+}\left(\theta_{s}\right)>x\right\} d x
$$


Hence

$$
\begin{aligned}
\rho_{+}^{-1}(s) & =\frac{1}{s} a p_{-}(s)=a \int_{0}^{\infty} e^{-s t} \mathrm{P}\left\{\xi^{-}(t)=0\right\} d t \\
& =s \int_{0}^{\infty} \int_{0}^{\infty} e^{-s t} \mathrm{P}\left\{\xi^{+}(t)>x\right\} d x d t \\
& =s \int_{0}^{\infty} \int_{0}^{\infty} e^{-s t} \mathrm{P}\left\{\tau^{+}(x)<t\right\} d x d t .
\end{aligned}
$$

Inverting (37) with respect to $s$ and using (4) we obtain

$$
\begin{aligned}
a \mathrm{P}\left\{\xi^{-}(t)=0\right\} & =\int_{0}^{\infty} \frac{\partial}{\partial t} \mathrm{P}\left\{\tau^{+}(x)<t\right\} d x \\
& =\int_{0}^{\infty} \frac{\partial}{\partial x} F(t, x) x t^{-1} d x=t^{-1} \mathrm{E}_{+} \xi(t)
\end{aligned}
$$

whence (32) follows.

We get $\mathrm{E} \xi^{+}\left(\theta_{s}\right)=\rho_{+}^{-1}(s)$ in both cases, and thus (36) implies (33). Inverting (33) with respect to $s$ and $\alpha$, we prove the first relation in (34).

In view of (37),

$$
\begin{gathered}
\mathrm{E} \xi^{+}\left(\theta_{s}\right)=s \int_{0}^{\infty} \int_{0}^{\infty} e^{-s t} \mathrm{P}\left\{\tau^{+}(x)<t\right\} d x d t \\
\mathrm{E} \xi^{+}(t)=\int_{0}^{\infty} \mathrm{P}\left\{\tau^{+}(x)<t\right\} d x
\end{gathered}
$$

Differentiating $\mathrm{E} \xi^{+}(t)$ we get

$$
d \mathrm{E} \xi^{+}(t)=\int_{0}^{\infty} \frac{\partial}{\partial t} \mathrm{P}\left\{\tau^{+}(x)<t\right\} d x d t=t^{-1} \mathrm{E}_{+} \xi(t) d t
$$

Substituting $d \mathrm{E} \xi^{+}(y)$ in the first line of (34) we prove the second relation in (34). The proof of the theorem is complete.

Considering the graph of the trajectory of the process $\xi(t)=S(t)-t$, an analogue of formula (32) is proved in [7] for the case of $\lambda<\infty$ (see Theorem 2.1 in [7]). Relation (32) also holds for upper semicontinuous processes $\xi(t)$ with $a>0$ if $\lambda=\int_{R} \Pi(d x) \leq \infty$. An analogue of relation (32) holds for lower semicontinuous processes with $a<0$ and $\lambda \leq \infty$. The corresponding result generalizes the Seal formula in this case.

Relation (31) for $x=-u$ defines the ruin probability in the bounded interval $[0, t]$ for the classical risk process

$$
\xi_{u}(t)=u+a t-S(t), \quad S(t)=\sum_{k \leq \nu(t)} \xi_{k}
$$

with the initial capital $u>0$ and $a>0$, where $\nu(t)$ is a Poisson process with intensity $\lambda$ and where $\mathbf{P}\left\{\xi_{k}>0\right\}=1$. This provides a relationship between the ruin probability

$$
\Psi_{t}(u)=\mathrm{P}\left\{\xi_{u}\left(t^{\prime}\right)<0 \text { for some } t^{\prime}<t\right\}=F_{-}(t,-u)
$$

for $u>0$ and the survival probability for $u=0$ defined by

$$
\bar{\Psi}_{t}(0)=1-\Psi_{t}(0)=(a t)^{-1} \int_{0}^{\infty} \bar{F}(t, x) d x
$$

in view of equality (32) if $m=\mathrm{E} \xi(1)>0$ and $\xi(t)=\xi_{0}(t)$. Using this notation formula (31) becomes

$$
\Psi_{t}(u)=F(t,-u)+\left.a \int_{0}^{t} \bar{\Psi}_{t-y}(0) F^{\prime}(y, x)\right|_{x=-u} d y .
$$


Since

$$
\mathrm{E}_{+} \xi(t)+\mathrm{E}_{-} \xi(t)=m t, \quad m=a-\lambda \mu_{1}, \quad \mu_{1}=\mathrm{E} \xi_{1}>0,
$$

the ruin probability $\Psi_{t}(0)$ can be rewritten by using $\mathrm{E}_{-} \xi(t)$ as follows:

$$
\Psi_{t}(0)=\frac{\lambda \mu_{1}}{a}-\frac{1}{a t} \int_{R^{-}} F(t, x) d x \underset{t \rightarrow \infty}{\longrightarrow} q_{-}=\frac{\lambda \mu_{1}}{a} \quad \text { for } m>0 .
$$

Note that there are two simple roots of the Lundberg equation,

$$
r_{1}(s)=-\rho_{-}(s)<0<r_{2}(s)=R_{+}(s)
$$

for a lower semicontinuous stochastic process (see Example 1) or for a lower almost semicontinuous process. The first of these roots determines the characteristic function of $\xi^{-}\left(\theta_{s}\right)$ by

or

$$
\varphi_{-}(s, \alpha)=\frac{\rho_{-}(s)}{\rho_{-}(s)+i \alpha}
$$

$$
\varphi_{-}(s, \alpha)=\frac{p_{-}(s)(b+i \alpha)}{p_{-}(s)+i \alpha}, \quad \rho_{-}(s)=b p_{-}(s) .
$$

The second root, $R_{+}(s)$ partially determines the characteristic function of $\xi^{+}\left(\theta_{s}\right)$, since a certain function $Q(s, \alpha)$ is involved in the expression above. In particular, the following result holds for the lower almost semicontinuous risk process $\xi(t)=S(t)-C(t)$ with random premiums and with the cumulant given by

$$
\psi(\alpha)=\psi_{+}(\alpha)+\psi_{-}(\alpha), \quad \psi_{-}(\alpha)=-\frac{\lambda_{2} i \alpha}{b+i \alpha}, \quad b>0, \lambda_{1,2}>0,
$$

where $\psi_{+}(\alpha)=\lambda_{1}(\varphi(\alpha)-1)$ is the cumulant of the claim process $S(t)$ and where $\psi_{-}(\alpha)$ is the cumulant of $-C(t))$.

Proposition. Let a process $\xi(t)$ have the cumulant given by (43). Then the characteristic function of $\xi^{+}\left(\theta_{s}\right)$ admits the representation

$$
\begin{gathered}
\varphi_{+}(s, \alpha)=\mathrm{E} e^{i \alpha \xi^{+}\left(\theta_{s}\right)}=\frac{s}{p_{-}(s)}\left[\left(R_{+}(s)-i \alpha\right) Q(s, \alpha)\right]^{-1}, \\
Q(s, 0)=s\left(p_{-}(s) R_{+}(s)\right)^{-1}, \quad Q\left(s, i \rho_{-}(s)\right) Q\left(s,-i R_{+}(s)\right) \neq 0 .
\end{gathered}
$$

If $m=E \xi(1)<0$, then $R_{+}(s) \underset{s \rightarrow 0}{\rightarrow} R_{+}>0$ and the characteristic function of $\xi^{+}$is given by

$$
\varphi_{+}(\alpha)=|m| b\left(R_{+}-i \alpha\right)^{-1} Q(0, \alpha)^{-1}, \quad Q(0,0)=\frac{|m| b}{R_{+}} .
$$

The main term of the ruin probability as $u \rightarrow \infty$ is determined uniquely by the exponent $e^{-R_{+} u}$ up to a certain factor $\kappa$, namely

$$
\Psi(u)=\mathrm{P}\left\{\xi^{+}>u\right\} \sim \kappa e^{-R_{+} u}, \quad \kappa \sim \mathrm{P}\left\{\xi^{+}>0\right\}=q_{+} .
$$

Proof. Since the denominator of the characteristic function of $\xi\left(\theta_{s}\right)$,

$$
\varphi(s, \alpha)=\frac{s(b+i \alpha)}{k_{*}(s, \alpha)}, \quad k_{*}(s, \alpha)=s(b+i \alpha)+\psi_{+}(\alpha)(b+i \alpha)-i \alpha,
$$

admits the representation

$$
k_{*}(s, \alpha)=Q(s, \alpha)\left(R_{+}(s)-i \alpha\right)\left(\rho_{-}(s)+i \alpha\right),
$$

we obtain the representation for $\varphi_{+}(s, \alpha)$ in view of the main factorization equality (1.64) in [3]. Relation (45) in the case of $m<0$ follows from (44) by passing to the limit as $s \rightarrow 0$ in the relation for $\varphi_{+}(\alpha)$. 
Note also that if the process $\xi(t)$ is semicontinuous, then most of the approximate relations for $\Psi(u)$ given in [3] (see relations (3.17), (3.23)-(3.25), and (3.44) therein) still hold. These relations are obtained by expressing the approximations $\kappa$ for the coefficient $q_{+}$and for $R_{+}$in terms of the second and third moments of $\xi(1)$.

\section{BIBLIOGRAPHY}

1. F. A. Spitzer, Principles of Random Walk, D. Van Nostrand, Princeton, 1964. MR0171290 $(30: 1521)$

2. F. A. Spitzer, A combinatorial lemma and its application to probability theory, Trans. Amer. Math. Soc. 82 (1956), 323-329. MR0079851 (18:156e)

3. D. V. Gusak, Boundary Problems for Stochastic Processes with Independent Increments in the Risk Theory, Proc. Inst. Math. Nat. Acad. Sci. Ukraine, vol. 67, Kyiv, 2007 (Ukrainian) MR2382816 (2009e:60002)

4. J. H. B. Keilson, The first passage time density for homogeneous skip-free walks on the continuum, Ann. Math. Statist. 34 (1963), no. 3, 1001-1011. MR0153060(27:3029)

5. V. M. Zolotarev, The moment of first passage of a level and the behaviour at infinity of a class of processes with independent increments, Teor. Verojatnost. i Primenen. 9 (1964), no. 4, 724733; English transl. in Theory Probab. Appl. 9 (1964), no. 4, 653-661. MR0171315 (30:1546)

6. A. A. Borovkov, On the first passage time for a class of processes with independent increments, Teor. Verojatnost. i Primenen. 10 (1965), no. 2, 360-364; English transl. in Theory Probab. Appl. 10 (1965), no. 2, 331-334. MR0182052 (31:6276)

7. S. Asmussen, Ruin Probabilities, World Scientific, Singapore, 2000. MR1794582(2001m:62119)

Institute of Mathematics, National Academy of Science of Ukraine, Tereshchenkivs'Ka Street, 3, 252601 KYIV-4, UKRAine

E-mail address: random@imath.kiev.ua

Received 10/DEC/2010

Translated by S. KVASKO 